(Aus dem anatomischen Laboratorium der psychiatrischen und Nervenklinik der Charité [Cieh. Rat Prof. Ziehen.])

\title{
Beitrag zur vergleichenden Anatomie der Substantia nigra, des Corpus Luysii und der Zona incerta.
}

Von

\author{
Dr. TORATA SANO.
}

(Hierzu 'Taf. I-II.)

(Fortset\%ung.)

\section{Schwein.}

Als einen Vertreter der Ungulaten habe ich das chinesische

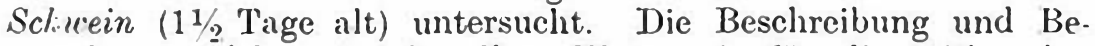
sprechung erfolgen in dersclben Weise wie für die seither besprochenen Säuger. Ich habe absichtlich ein junges 'Tier, dessen IIarkscheidenbildung noch nicht vollendet ist, gewählt, weil es die hier in Betracht kommenden morphologischen Verhältnisse besonder's deutlich \%eigt.

Der Schnitt $31^{1}$ (vergl. Fig. 33) schncidet ventral das vordere Brückenriertel.

D) is Substantia nigra findet sich hier ventrolateral vom lateralen Areal der medialen Schleife, dessen Fasern hier bereits im Aufsteigen zum Yordervierhügel begriffen sind. Sie hängt clorsal mit dem Corpus parabigeminum zusammen. Lateral von ihr verlaufen die Fasern des von Hatscliek mit $\mathrm{v}$ be\%eichneten Bündels. Ihre Dicke beträgt $1, t \mathrm{~mm}$. In ihrem weiteren Verlaufe gelangen die $v$-Fasern in das Brückengrau. Der Faser\%ug $\mathrm{m}$ ron Halschek ans dem Corpus parabigeminum in clie Haube ist eben angedeutet. Dorsalwärts steigt stielartig der Faserzug d von Hutschek zur Vordervierhügelschleife auf.

Das sehr unscharf begrenzte, dorsomedial rom Corpus parabigeminum liegende graue Feld entspricht wohl dem Feld II der anderen Tiere.

Der Fuss grenzt jetzt ummittelbar an die Verbindungsbrücke der beiden Areale der medialen Schleife und ist von letzterer dank seiner schwächeren Tinktion leicht $\%$ u trennen.

Wenn man die eben erwähnte Verbindungsbrücke cerebralwärts verfolgt, so ergibt sich, dass ihre Fascrn teils ventrolateral in den Fuss einbiegen, teils der Vordervierhügelschleife nachiiehen. Spinalwärts verbleiben ihre Fasern in der medialen Schleife.

tus dem Fuss ziehen Fasern anscheinend dorsolateral und gesellen sich der Vordervierhügelschleife $\%$. Freilich muss ich dahingestellt lassen, ob es sich nicht vielleicht doch nur um 
eine voriubergehende Vermischung der Fasern des Fusses mit der medialen Schleife handelt.

Das Sprizkasche Biindel, d. h. das Bündel von der Schleife \%um liuss, ist auch beim Sichwein mur sehr schwach entwickelt. Immerhin sieht man auf den folgenden Sichnitten einige liasern, die dem Verlauf dieses Bïndels entsprechen.

Das Briticliengrau medial rom Fuss sendet beiderseits Fortsät\%e aus. Der laterale Zapten (Npl Z) schiebt sich \%wischen das mediale Areal der medialen sichleife und den F'uss; er ist von der Substantia nigra noch weit getrennt.

Jin Schnilt, welcher ventral das rordere Briickenfiinflel schneidet (329), zeigt die Sulsstantia nigra bereits erheblich grösser. Hhre Jicke beträgt nur $1,1 \mathrm{~mm}$. Medial wird sic rom lateralen Areal der medialen Schleife, ventral und lateral von dem allmählich sich formierenden Fuss, dorsal \%um Teil rom Faser\%ug m ron Halschek: begrenzt. Sie grenct ausserdem dorsoliteral an das Corpus parabigeminum. Sie besteht noch im allgemeinen überall aus kompakter grauer Substan\% und längsgetroffenen Fasern.

Der Schnitt $3 t^{3}$ (vergl. Fig. 3t) schneidet ventral noch den verdersten Briickenrand und die Bindearmkreu\%ung.

Die Substantia nigra ist grösser geworden. Hure Jicke beträgt $2,4 \mathrm{~mm}$. Sie zerfällt schon cleutlich in eine schmale ventrale Zona recticulatia und eine breite Zona compacta.

Das Corpus parabigeminum ist etwas kleiner geworden. Der Faserzug $v$ ist gan\% verschwunden, der Fascr\%ug d \%eigt \%iemlich diffus angeordnete Fasern, und der Faser\%ug $\mathrm{m}$ ist auf rereinzelte Fasern reduriert.

J)ie Retikulation der Zonal reticulata ist anscheinend, Wonigstens zum 'Teil, dadurch \%ustande gekommen, dass die im rorigen schnitte e.r. ähnte Fasermasse im dorsolateralen Teile des Fusses nun von grauen Balken in Bündel abgeteilt wird. Diese Bïndel zeigen noch die frühere schräge Verlautsrichtung ihrer Fasern. Sie hestehen aus Fasern, die aus der medialen Schleife stammen, und werden schliesslich dem Fuss einverleiht. Sic sind also dem Gesamtrerlauf nach mit den Bündeln identisch. die von Flechsig als Fussschleife beschrielsen worden sind. Während abor die Bündel der Flechsigschen Fussschleife bei vielen anderen Näugern eine durch gratue Ballien isolierte Bündelgruppe bilden, die erst viel später dem Fuss cinverleibt wird, sind unsere Bündel hei dem Shehwein viel weniger selhständig. Sie entsprechen, wenn man den Vorgang bei der lintstehung der Bündelgruppe und den Verlauf der Bïndel berücksichtigt, in vielen Bezichungen eher der Bündelgruppe (; der früher besprochenen Tiere als der Fussschleife von Flechsig. Ich will also diese ganze Bündelgruppe: bei der Beschreilung und Besprechung der folgenden schnitte unter aller Reserve als Bündelgruppe C' bezeichnen.

Im lateralsten Teil der Kona reticulata sieht man einige schrïgr geschnittene feine Fasern bei Strim, die wohl als eine Andentung des Stratum intermedium aufgefasst werden lïnnen. 
Die mediale Partie der Zona compacta wird grösstenteils von den wurelartig aufsteigenden Schleifenfasern verdeckt.

Der laterale Zapfen des Brïckengraus hängt jet\%t direkt mit einem schmalen, auf der Figur durch ein Kreuz bezeichneten Nahtstrcifen grauer Substanz, der der Substantia nigra \%u\%urechnen ist, \%usammen; der mediale Zapfen fliesst mit der Substantia perforatia posterior \%usammen.

Das Feld M findet sich dorsomedial vom Corpus parabigeminum, ist aber unscharf begrent. Es geht allmählich in die Substantia. nigra über.

Das Spitzkasche Bündel ist bereits schwach entwickelt.

Auf einem Schnilt (36'), welcher noch etwas weiter proximal liegt, misst die Substantia nigra bereits $2,7 \mathrm{~mm}$ im Dickendurchmesser. Sie geht medial unterhall des medialen Areals der medialen Schleife in die Substantia perforata posterior iiber. Der Zusammenhang der Substantia nigra mit dem Grau der Haube durch grane Bällichen, velche zwischen den Bündeln der medialen Schleife aluftreten, ist auf Objektträger $35^{1}$ schon eben $\% u^{2}$ erkennen. Das Corpus parabigeminum ist hier schon viel kleiner geworden und in das Areal des Fusses eingebettet. Die Zona reticulata substantiae nigrae ist breiter geworden. Die Bündelgruppe $C$ ist sehr mächtig entwickelt; in der lateralsten und in der medialsten Fusspartie ist dic Retilulation nicht so deutlich wie in der mittleren. Das Stratum intermedium, das noch immer nur in der lateralsten Partie der Zona reticulata \% sehen ist, ist etwas deutlicher geworden.

In der Zona compacta sieht man schon eine Andeutung eines Geflechtes, das dem Geflecht $D^{1}$ der seither besprochenen säuger entspricht. Es hängt mit den Fibrac efferentes tecti dentlich \%usammen. In der lateralen Partic sieht man viele Fasern, die sich der lateralen schleife zugesellen. Viele der eben besprochenen Fasern in der Zona compacta viehen anscheinend durch das Maschenwerk der Zona reticulatio in den Fuss.

Der Schnill 39 (vergl. Fig. 35) schneidet ventral die Wur\%elbündel des Oculomotorius. In der mittleren Partic ist der rote Kern getroffen.

Die Sulstantia nigra ist sehr mächtigg entwickelt; sie ist in der Form etwa länglich oval. Ihre Jicke beträigt $3,6 \mathrm{~mm}$. Der grösste 'Teil der Substantia nigra wird von der Zona compacta gebildet.

In der Zona reticulata ist die Bündelgruppe C etwas lileiner geworden und findet sich immer noch hatutsächlich in der mittleren Partic. Im lateralen Abschnitt des Fusses ist ein Processus lateralis eben angedentet.

Das Stratum intermedium ist nur im Processus lateralis eben zu erkennen.

In der Zona compacta sieht man sehr reichliche Fascrn, und zwar \%wei deutlich differenzicrte Fasergeflechte. Das laterale ist das Geflecht $\mathrm{D}^{\prime}$; das mediale entspricht dem Cieflecht . $)^{\mathrm{m}}$ der 
früher besprochenen Säuger. Beide Geflechte bestehen hauptsächlich aus Fibrae efferentes tecti. Ihre Fisern \%iehen weiterhin durch das Maschenwerk der Zona reticulata, um sich im Fuss zu verlieren.

Das Corpus parabigeminum ist verschwunden.

Der Pedunculus corporis mamillaris, der \%uerst auf Objektträger $39^{1}$ auftritt, ist sehr mächtig entwickelt und trennt die Substantia nigra ziemlich scharf ron der Substantia perforata posterior.

D)as Bündel der Substantia nigra zum Pedunculus corporis mamillaris war auf Objektträger 36 und $37 \%$ sehen.

Das Pedamentum laterale ist ventrolateral rom Pedunculus corporis mamillaris deutlich zu sehen. Einige Fasern aus der Haube, die wohl nicht zum 'Tractus peduncularis transversus \%u rechnen sind, treten in das Pedamentum laterale cin. Das Feld M ist. dorsolateral ron der Substantia nigra als ein immer noch unscharf begrenztes Feld \%u sehen.

Der Schnitt $45^{4}$ (vergl. Fig. $3(i)$ schneidet ventral den spinaleren Teil des Corpus mamillare und das Meynertsche Bündel.

Die Substantia nigra erreicht ihr IIaximum. Ihre Dicke beträgt $4,8 \mathrm{~mm}$. In der Zona reticulata zeigt die Bündelgruppe (" cine sehr lockere Bündelanordnung.

Der Fuss ist in der medialen Hälfte äusserst schmächtig geworden, hat hingegen im lateralen Drittel stark zugenommen. .is bildet hier ein sehr charaliteristisches stumpfwinkliges Dreieck.

Der Processus lateralis ist noch undeutlicher geworden als im vorigen Schnitte. Das Stratum intermedium ist katum \%u sehen. In der 'Zona compacta sind die Geflechte $\mathrm{I}^{\prime}{ }^{\prime}$ und 1$)^{\mathrm{m}}$ jet\%t miteinander verschmolzen.

Ventral vom roten Kern sieht man eine Zone ron Fasern dorsolateraler Richtung, die auf Objelitträger 43 \%uerst auftritt und auf dem hier abgebildeten Schnitt riemlich breit ist. Sie entspricht der halbmondförmigen Schicht der seither besprochenen Säuger HmSch.

Ein Faser\%ug, der dem Faser\%ug B der früher besprochenen Säuger entspräche, ist nicht \%u sehen.

In der Gegend des (ieflechtes $D^{1}+D^{m}$ sieht man eine Bündelgruppe. dic auf der Figur mit W bezeichnet ist. Wenn man cliese Bündel spinalwärts (vergl. Figg. 34 und 35 ) verfolgt, so ergibt sich ein sehr interessanter Verlauf: die Querschnitte der Bündel verschieben sich nämlich mehr und mehr in dorsolateraler Richtung. Jabei passieren sie das Areal der Vordervierhügrelschleite und liommen schliesslich mitten in das Haubengebict \%u liegen. Eine noch weitere Verfolgung gelingt nicht mit Sicherheit. C'erebralwärts gelangen sie in den Fuss.

Dieser Befund erscheint mir insofern äusserst interessant, als er vielleicht gecignet ist, auf die \%entrale Bahn der motorischen Hirnnerven Licht zu werfen. Bekanntlich ist man jetzt geneigt, 
anzunehmen, dass die Bahn der motorischen Hirnnerven (corticobulbäre Bahn) im Fuss medial von der Pyramidenbahn liegt, dann aber im Bereich der Briicke in die mediale Schleife eintritt, und \%war als sogenannte Fussschleife flechsigs (laterale pontine Bündel von Schlesinger), um schliesslich auf unbekanntem Wege. zu den Kiernen der motorischen Firnnerven $\%$ gelangen. Śperiell haben Bech(eren $\left.{ }^{1}\right)$ und Hoche ${ }^{2}$ ) diese Anschauung vertreten. während Schlesinger's) bekanntlich seine lateralen pontinen Bündel als sensibel aufgefasst hat. Meine Befunde bei dem Schwein scheinen sehr darauf \%u deuten, dass die gesuchte Bahn der motorischen Hirnnerven in den $\mathrm{W}$-Bündeln gegeben ist. Man wird dann freilich scharf betonen müssen, dass es sich, im Gegensat\% zu den Bündeln Flechsigs und Schlesingers, um Bündel handelt. die niemals einen Teil der Schleife bilden, sondern das Schleifenareal nur passieren. Ich möchte zur Unterstït\%ung meiner Anschauung noch darauf aufmerksam machen, dass belianntlich die Fussschleife sich von den übrigen Fasern der medialen Schleife durch ihre hellere Farbe unterscheidet, und dass gan\% dasselbe Unterscheidungsmerkmal auch den von mir beschriebenen W-Bündeln \%ukommt.

I)er Pedunculus corporis mamillaris hat sich mehr in die Breite ge\%ogen.

Der 'Tractus peduncularis transversus tritt zum erstenmal deutlich auf Objektträger 42 , zwischen dem Fuss und dem Pedamentum laterale, auf. Er \%eigt sich hier auf dem Sichnitt ti) als ein riemlich mächtiges Bündel.

Das Feld $\mathrm{M}$ ist noch riemlich gross und findet sich dorsolateral vom Fasergeflecht $\mathrm{D}^{\prime}+\mathrm{D}^{\mathrm{m}}$.

A uf einem Schnilt $\left(50^{2}\right)$, welcher ventral das Corpus mamillare in Bereich des Austritts des Fasciculus mamillaris princeps schneidet, ist die Substantia nigra ctwas kleiner geworden. Ihre Dicke beträgt $4,6 \mathrm{~mm}$. Von allen Teilen, besonders aber dem lateralen 'Teil der Zona reticulata substantiae nigrae dringen viele graue Balken in clen Fuss ein. Sie entsprechen den friiher besprochenen A-Feldern. In der Zona compacta substantiae nigrae sind das Fasergeflecht $\mathrm{D}^{\prime}+\mathrm{D}^{\mathrm{m}}$ und die Bündelgruppe $\mathrm{W}^{\circ}$ viel weiter ventral gerüclit.

Die Faser\%iige ' $I$ ' und $T$ ' habe ich nicht mit Sicherheit identifizieren können. Aus der Gegend der halbmondförmigen Schicht ziehen Fasern ventral in den medialsten Fussteil. Sie entsprechen den Fasern des Büschels $\mathrm{K}$ der scither besprochenen 'T'iere (sehr' deutlich auf Objektträger $4 \bar{i}$, \%uerst auf (Objelitträger 43 ).

Ein graues Feld, das der Substantia reticulata lateralis pedis entspricht, ist bei dem schwein nur andeutungsweise vorhanden.

1) Bechtcrew, Leitungshahnen im Gehirn und Rïckenuark. Jeiprig 1899. S. 318 .

2) Hocke. Arch. f. Psych. Bd. XXX. S. 103.

2) Schlesinger, Arbeiten aus dem neurolog. Inst. der Wiener Inivorsität. Heft IV, s. s... 
Die bemerlenswerteste Veränderung auf diesem Schnitte ist das Auftreten des Feldes $\mathrm{H}$ von Forel, das schon ant Objekt.träger $49^{3}$ sichtbar ist. Deutlich aus dem roten Kern stammend, reicht es bereits riemlich weit lateral. Ein 'Teil seiner Fasern gelangt bis in ein Feld naho der medialen P'artie der Markiapsel des C'orpus geniculatum mediale, das vielleicht dem Feld Y der Katze entspricht

Der Schnith 52' (vergl. Fig. 37) schmeidet ventral noch das ricy d'Azyrsche Bündel bei seinem Austritt aus dem Corpus mamillare.

Die Dicke der Substantia nigra beträgt noch $1,6 \mathrm{~mm}$. Ihr retiliuliertes Aussehen ist jet\%t hauptsächlich durch dic Bündelgruppe $W^{\prime}$ bedingt, die jet\%t im Begriff ist, dem Fuss einverleibt z.u Werden. Der Rest des Geflechtes $\mathrm{D}^{1}+\mathrm{D}^{\prime \prime}$ ist zwischen den IV-Bündeln und den Fussbündeln noch sichtbar, ebenso die Fasern des Büschels $\mathrm{IK}$.

Die A-Felder sind viel rentraler geriickt.

Das Corpus Luysi: das aut (O)jektträger $51^{3}$ schon angedeutet ist, tritt hier deutlich auf. Es ist noch gan\% schmal. Seine Dicke beträgt $0,3 \mathrm{~mm}$. Die ventrale Markliapsel ist schwach, die dorsale Markliapsel ist gut entwickelt. Sic besteht a us Fasern des Feldes $\mathrm{H}^{2}$, \%u denen auch Fasern liommen, dic in den rorausgegangenen ichnitten, dorsal von $D^{1}+D^{m}$, \%u sehen waren (Vorläufer des Feldes $H^{2}$ ?). Die Fasern ziehen weiter deutlich in den Fuss.

Das Feld $\mathrm{H}^{1}$ ist sehr mächtig. Seine Fasern gehen lateralwärts grösstenteils lionvergierend in die Lamina medullaris lateralis über.

Aus dem Feld $\mathrm{H}^{2}$ zichen 1. sehr spärliche \%erstreute Fasern dorsolateral gegen die Lamina medullaris lateralis. Wegen des zerstreuten Verlautes dieser Fasern \%erfällt die Zona incerta bei dem Sichwein überhaupt nicht scharf in eine mediale und eine laterale Abteilung; 2. Fasern als ein riemlich geschlossenes Bündel lateralwärts durch die Zona incerta, um zum 'Teil an der lateralen Seite des Fusses im Bogen in den Tractus opticus ïberzugehen, heror die Meynerlsche Kommissur auttritt; sie sind mit den Fasern identisch, die aus der dorsalen Markkapsel des C'orpus Luysii (diese Markkapsel besteht bei den seither besprochenen 'Iieren hauptsächlich aus Fasern des Feldes $\left.H^{*}\right)$ durch den Fuss an die rentrale Seite des Fusses gelangen und in den 'Jractus opticus iibergehen. Dieses Bündel wird vom l'uss resp. vor dem Fuss durch eino graue, sehr faserarme, bogenförmige Zone, die auf (ler Figur mit i bezeichnet ist, getrennt. Der dorsale Abschnitt dieser Zone (id auf Fig. 3s) kamn wohl mit Recht als ein besonderer 'l'eil der Zoral incerta aufgefasst werden. Er entspricht jenem 'l'eil der Zona incerta beim Menschen, der durch den lateralen Fortsat\% des Feldes $\mathrm{H}^{2}$ von der $\mathrm{Hau}^{2}$ tmasse der Zona incerta getrennt wird. Bci dem Menschen ist der Unterschied des Fasergehaltes in beiden 'Teilen nicht so gross wie bei dem Schwein. Bei dem Macacus, Lemur und bei der Katze tritt der laterale lortsatz des Feldes $\mathrm{H}^{2}$ nicht als ein Bündel auf, sondern in ciner gan\% \%er- 
streuten Faseranordnung, so dass man die eben erwähnten Abteilungen nicht unterscheiden kann. Der ventrale Abschnitt liamn iibrigens auch als ein 'Teil der jet\%t sich formierenden Gitterschicht aufgefasst werden, in welche greichtalls einzelne Fasern des Felcles $\mathrm{H}^{2}$ gelangen.

Dejerine ${ }^{1}$ ) bestreitet die Existen\% der von stilling beschriebenen und nach ihm genannten Fasern, d. h. der Verbindungen wwischen den Fasern der Markiapsel des Corpus Luysii und dem Tractus opticus. Wenn man aber das Bild der Figur 37, das die direkte Verbindung des Feldes $\mathrm{H}^{2}$ und des 'Tractus opticus darstellt, vor Augen hat und gleich\%eitig an die wesentliche Beteilisung der Fasern des Feldes $\mathrm{H}:$ an der Bildung der dorsalen Markkapsel des Corpus Juysii bei den seither besprochenen Säugern denkt, so liamn man nicht wohl an der Existenz von Fasern wweifeln, die von der dorsalen Markliapsel des Ciorpus Luysii durch den Fuss in den Tractus opticus gelangen.

Die Zona incerta ist breit. Sie geht medialwärts in die Zona transitoria und lateralwärts in die beginnende Gitterschicht ïber.

Das Pedamentum laterale ist seit Objektträger j̄l nicht mehr \%u sehen.

Der Schnitt j.t' (vergl. Fig. 38) schneidet ventral noch das aufsteigende Vicy d'dzy:sche Bündel.

Die Substantia nigra ist seit Objelitträger 'j $t^{\prime} \mathrm{im}$ wesentlichen verschwunden. Im medialsten Fussabschnitt sind allerdings noch ein\%elne graue Felder erkemnbar, die als Substantia reticulata medialis pedis bezeichnet und eventuell auch als Rest der Substantia nigra gedeutet werden können.

J)as Corpus Luysii ist viel grösser geworden. Sein lateraler 'Teil wird besonders scharf begrenzt. Seine Dicke beträgt 1, $(1 \mathrm{~mm}$. Die dorsalo Markkapsel ist viel deutlicher geworden. Die ventrale Markliapsel ist sehr schwach entwiclielt und liegt ummittelhar dorsal vom Fuss.

Man sieht eine ganz leichte Einkerbung im Fuss an der lateralen Grenze des Corpus Luysii. Sie entspricht der beim Menschen und bei der Katze beschriebenen Einkerbung $\mathrm{N}$.

Im Fuss sind ausser den A-Feldern seit Objelitträger ‘)3 viels graue Balken aufgetreten, die mit dem Grau des ('orpus Luysii zusammen zu hängen scheinen. Diese grauen Balken, die auf der Figur mit i" bezeichnet sind, diirfen jedenfalls nicht zur sub)stantia nigra gerechnet werden. Die A-Felder liegen in ganzen ventral ron ihnen.

Die Zona transitoria ist breit. Sie schiebt sich \%wischen den medialen Ursprungsteil des Feldes $\mathrm{H}^{2}$ und die dorsale Markiapsel des Corpus Luysii und geht in die Zona incerta, und \%war in die dorsal rom Fuss liegende faserarme Zone allmählich iiber.

1) Dejerine, Anatomio des centres nerveux. Paris 1901. 13cl. 2. 
Der Schnill 573 (vergl. Figr. 3!)) schneidet ventral den let\%ten Rest des aufsteigenden Fasciculus mamillaris princeps und den spinalen 'Teil des 'Tuber cinereum.

D)as ('orpus Luysii ist kleiner geworden. Seine Dicke beträgt nur noch (1, $8 \mathrm{~mm}$. Die dorsile Markkapsel tritt eher noch sehärfer hervor, während die ventrale Iarkkapsel noch schwächer geworden ist. Shillingsche Fasem sind kaum noch zu sehen.

Die A- und A"Felder sind miteinander verschmol\%en und verbinden sich basalwärts zu einer gratuen Leiste, wie sie uns auch bei anderen Süugern begegnet ist. Diese grauc Leiste muss vom Ganglion infrapedunculare von G'uner merschieden werden, denn dieses entwickelt sich allmählich im ventralen Fussabschnitt selbst, wäbrend jene sich im Zusammenhang mit dem Grau der Substantia nigra und des Corpus Luvsii ans den A- und $A$ "-Feldern entwickelt und erst nach und nach in den ventralsten Fussab. schnitt gelangt. friiher.

J)ie Substantia reticulata medialis pedis rerhält sich wie

Die Zona incerta geht in die jeťt gut ausgebildete Gitterschicht ïber.

Die Zono i zerfällt jetzt nach der dorsolateralen V(erschiebung lateralsten Fussteils in einen dorsalen Abschnitt id und einen ventralen Abschnitt iv. Die 'Teilung ist a uf Objektträger 5.5' schon angedeutet. Einen scheinbar ähnlichen Vorgang der 'T'eilung haben wir nur bei dem 1 Ienschen (verol. Schnitt 171) gesehen; es handelt sich aber dort $\mathrm{mm}$ die Zweiteilung des hakenförmigen Feldes in den eigentlichen Haken und die begimnende 'Zona incertal, hier um die Zweiteilung eines besonderen Feldes i, das teils der Zona incerta. und teils viclleicht der Gitterschicht angehört.

Der dorsale Abschnitt id rerhält sich gegen die Umgebung wie friiher. 1)er ventrale Abschnitt iv \%eigt einerseits Fasern aus dem Feld $\mathrm{H}^{*}$, die in den Tractus opticus übergehen, andererseits Fasern der Meynertschen Kommissur, die der ventralen Flïche des Fusses entlang verlauten, um schliesslich unter dorsomedialer Umbiegung in der medialsten Fusspartic (ventromedial rom Corpus Luysii) \%u endigen. Wenn man mit diesem Befunde die entsprechenden Partien des Menschen-, Macacus-, Lemur- und Katzengehirns vergleicht, so gelangt man $\% u$ dem äbereinstimmenden Resultat, dass 1. Fasern der Meymertschen Kommissur in dorsomedialer Umbiegung in den medialsten Fussteil und das benachbarte Grau ( + auf Fig. 40) riehen und in spinaleren Ebenen anscheinend auch in das basale längsbündel äbergehen, dass 2. rom Feld $H^{2}$ Fasern durch das Corpus Luysii oder direkt in die Meynertsche Kommissur gelangen (Mensch 465, 466, 472, 475, 493; Lemur 45--5̆; Macacus 151, 148, 145, 141, Kat\%e 180, $218-225)$.

I)ie Zona transitoria ist noch deutlich zu sehen. 
Das auf der Figur mit Z bezeichnete Feld entspricht dem Feld $Z$ der Katt\%e.

Der Schnill 5:9² (vergl. Fig. 40 ) schneidet ventral den cerebralen 'Teil des 'luber cinercum.

Das Corpus Luysii ist im Begriff \%u verschwinden. Es ist nicht mehr als ein einheitliches Feld zu sehen. Es stellt im wesentlichen nur noch einige \%wischen den Fussbündeln zerstreute graue Inseln dar, die mit den verschmolzenen A- und A'-Feldern \%usammenhängen. Ton der ventralen Markiapsel ist nichts mehr ïbrig geblieben, der Rest der dorsalen Markkapsel ist noch zu seluen.

Die Felder $\mathrm{H}^{1}$ und $\mathrm{H}^{2}$ sind schon sehr faserarm. Die laterale Partie des Feldes $\mathrm{H}^{2}$ hat sich fast erschöpft, die mediale Partie zeigt eine sehr diffuse Faseranordnung und fliesst mit dem Feld $\mathrm{H}^{1}$ zusammen. J)abei sicht man cinige Fasern aus der medialen Partie des Feldes $\mathrm{H}^{2}$ ventromedial an der medialen Seite der Columna fornicis ventralwärts ziehen (Fortsatz $x$ von Lülliker).

Die Zona transitoria ist viel lileiner geworden. Sie geht noch immer in den Alschniit id über, von dem sie sich durch ihren Fiscrreichtum unterscheidet.

Der dorsale Abschnitt der Zone i, d. h. id, verhält sich wie früher. Der ventrale Abschnitt iv \%erfällt jet\%t in \%wei T'eile, einen lateralen, zwischen dem Fuss und dem 'l'ractus opticus liegenden, und einen medialen, mit den $A^{\prime}$ - und A-Feldern verschmolzenen (jvil und ivm).

Auf den folgenden Schnitten verschwindet das Corpus Luysii mitsamt seiner dorsalen Narkiapsel sehr hald. Im medialen Fusstcil bleibt die Sulstantia reticulata medialis pedis, die sich etwas verdichtet, noch längere \%eit sichthar.

Der Fortsat\% $x$ von Killiker stammt vom Feld $\mathrm{H}^{2}$ und zicht sehr deutlich in die Forelsche Lommissur, dorsal von der Meynert. schen Kommissur.

Das Feld id verschwindet auf Objektträger 60. Das Feld is lässt sich lateralwärts, wenn man von dem Durch\%ug der Fasern der Meynerischen Kommissur abstrahiert, bis zur Spitze des Globus pallidus verfolgen. Medialwärts lässt sich das Feld iv noch längere Zeit als ein sehr schmaler Streifen bis zu dem Grau des Tuber cinereum, oberhalb des Chiasmas, verfolgen.

(Fortset\%ung folert.) 